\title{
Research on Product Design Teaching Based on Bionic Form Deduction
}

\author{
Sun Binbin
}

Beijing Normal University, Zhuhai, China

\author{
Keywords: Product design; Bionic; Form deduction; Course teaching
}

\begin{abstract}
This paper introduced the related theory of bionic design, the important and difficult problems and teaching reflections in course, combined with teaching practice, summarized the method and process of bionic design, so as to make this course easy to understand and efficient, and to provide reference for the product design major course teaching.
\end{abstract}

\section{Introduction}

The essence of the bionic design is to learn from the nature. Everything in nature has experienced the test of survival and evolution. Both in terms of aesthetics and structure, it has natural advantages, such as the screw with a golden section curve, the honeycomb structure with stability and order aesthetics, etc. Human beings use their learning ability and creativity to learn from nature and seek the best fit point between products and biology, social production activities and all nature.

The research object of bionic design is the natural objects "form", "color", "sound", "function", "structure", etc. applies the characteristic principle selectively in the design process, provides new ideas for design activities. From the attribute of bionic content, it can be divided into form bionic, functional bionic, structural bionic, texture bionic, color bionic, image bionic, etc. Form bionic is the most widely used and common way in product design, through abstracting and generalizing the characteristics of the typical things artistically, and through product semantics to express the emotional design features, brings users the potential sense of familiarity, psychological identity and pleasant use experience, and improves the added value of product.

\section{Teaching Analysis}

\subsection{Teaching aims}

Form bionic as the enlightenment of product modeling design, the main goal is to help students to build macro form thinking ability, understand to think and control the modeling as a whole, and apply bionic design method to practice flexibly, so that students can preliminarily acquire the modeling ability of product design. On the contents of ability training, it including : the modeling ability of element extraction and form deduction , artistic and creative abstract thinking ability, the spatial imagination ability of $2 \mathrm{~d}$ images to transform $3 \mathrm{~d}$ entities, overall control ability of form transformation and product design application, etc.

\subsection{Key point in teaching}

The emphasis of bionic design is to grasp the essential features of bionic things. Whether we start from the part or the whole, we must explore the form features that can best reflect the essence of bionic things in repeated design practices. After several times of abstraction and generalization, the elements and product shapes can be reasonably and skillfully combined and reflect: " be alike in spirit, but does not like the shape", which means that we can see that it is like something, but not too concrete and direct; At the same time, the bionic form should be able to adapt to the original functions of the product, or can improve product function, or to create new function, and through the bionic form to reflect a product semantics, then strengthen the product and people's emotional exchanges. It's important to note that, the application of form should not lead to defects in product 
use, it is not advisable to sacrifice the practicality of the product in order to achieve a certain bionic shape.

\subsection{Difficult point in teaching}

The difficulty of product form bionic is element abstraction and product application. Element abstraction requires designers to have strong creative thinking and painting skills, while product application requires logical analysis ability and overall grasp ability. In this process, there is no formula and principles, according to the author's own aesthetic experience and modeling ability, rather than simple imitation, to refine and re-create, and to design a modern product with industrial aesthetic feeling and suitable for mass production.

\subsection{Specific problems in teaching}

According to the author's teaching experience of bionic design courses in recent years, the following problems are summarized: 1 . Abstract methods cannot be embodied. The abstract process itself is a creative process, there is no absolute method and formula. In the existing books and online resources, there is no systematic form bionic design method theory to assist classroom teaching, only the ready-made design case can be used as a reference, as a result, students have a hard time comprehending, and each student has an uncontrollable degree of mastery; 2. The application of the element is too straightforward. Learn bionic design at the beginning, most students directly apply the selected element forms to the products, without several abstract steps, resulting in the product modeling language too concrete, simple and vulgar; In the selection of elements, there is a lack of thinking about the connection between bionic objects and design objects, and a kind of thing is arbitrarily applied to product modeling, resulting in inappropriate product semantics. 3. Lack of grasp of product practicality. After applying bionic forms and creating new shapes, instead of creating new functions, the basic functions of products are hindered, and there is no best connection between beauty and function.

\section{Teaching procedures}

\subsection{The general process of form bionic design}

(1) Determine the design object: What product are we going to design?

(2) Analyze product semantics and find suitable bionic objects: Guide students to find things that they are interested in from nature. In addition to studying the similarity in the form features, we should also consider the semantic connection of products. Through the "facial expression" of product bionic, to strengthen emotional communication between products and people.

(3) Recording and drawing the bionic objects: In order to understand and study the form of bionic objects, we should draw it in various angles, features and types, At the same time, we should collect literature extensively, in order to learn more about bionic objects.

(4) Form deduction: This step focuses on abstracting and extracting biological features. If this step is not done well, the final product design will often be too simple or too concrete, and the form is simple and not advanced. As shown in Figure 1, Through divergent thinking and artistic re-creation, with exaggeration, deformation, simplification and other methods, it abstracted and split the local features or overall features of bionic objects many times, then combined with the aesthetic form elements, to obtain a rich variety of abstract graphics.

(5) Product analysis: Analyze the main functions and ancillary functions of the product and consider which functional components and details are necessary, what kind of shape to carry these functions? at the same time, the influence of man-machine factors on product form should also be considered, and so on.

(6) Graphic apply and product design: According to the preliminary product application of abstract graphics, the following questions should be considered: How can the resulting abstract graphics be skillfully combined with the main functional components of the product? Function determines form, how can abstract graphics continue to evolve to accommodate product 
functionality? Can new product styling create new functionality?

(7) Program deepening and determination: Take into account the users, functions, ergonomics, usage scenarios and other factors of the product, to find an appropriate combination point of the bionic correlation, the beauty of the shape and the rationality of the function, then refine and deepen the initial product bionic form to obtain the final product modeling continuously.



Figure 1. The form deduction of the unicorn

\subsection{The teaching example: Form deduction - "from wolf to car design"}

The bionic design method is widely used in automobile design, attractive appearance is an important factor influencing the success of a car in the market, and the intuitive visual effect of bionic form can bring good market benefit and user empathy.

Design idea: As shown in Figure 1, in this case, the wolf was selected as the bionic object of the car, mainly based on the similar association of the dynamic characteristics and head characteristics of wolves. First of all, the designer first described wolves from different angles and poses, with emphasis on dynamic and head characteristics analyzing. On the one hand, the dynamic curve of the jumping posture of wolf is applied to the car body design; on the other hand, the front and side profile curves are extracted from the head shape of the Wolf and applied to the front face design of the car. In this way, the characteristics of " sporty" and "power" of wolf are integrated into the temperament of the car, adding the sense of potency and speed to the overall design of the car, presenting a strong visual effect and sense of wholeness.



Figure 2. Car bionic design based on wolf form deduction

\section{Conclusion}

Bionic design method is an important content in the undergraduate course teaching of product design major, the author has been teaching product bionic design course for many years, combining 
with the design project to carry out the course teaching practice, discussing the specific bionic design methods and procedures, to guide students to complete a complete process of form bionic design. The final teaching result can reach the expected goal basically, the student gains a lot from the design practice of this course.

\section{Acknowledgement}

Beijing Normal University, Zhuhai teachers' research capability promotion program Item no. 201850008

\section{References}

[1] Janine M. Benyus. Biomimicry-Innovation Inspired by Nature [M]. William Morrow Company, 2002.

[2] Sun Ningna, Dong Jiali. Bionic Design[M]. Changsha:iLEp:Hunan University Press, 2010.

[3] Jiang Na, Yang Junshun, Application of bionic in product design[J]. Packaging Engineering, 2006, 27(6):306-312.

[4] Yang Liying, Sun Ningna, Zhang Wenli. Bionic Design Flow from Natural Organisms to Product[J]. Packaging Engineering, 2010, 31(12):15-18.

[5] Tian Jun. Nature, Source and Direction: Luigi Colanis Bionic Design[J]. Zhuangshi, 2013(4): 35-40. 